$\mathrm{UW} / \mathrm{PT}$ 03-03

BUHEP-03-02

\title{
Little Inflatons and Gauge Inflation
}

\author{
David E. Kaplan ${ }^{a, c *}$ and Neal Weiner ${ }^{b, c \dagger}$ \\ a Department of Physics and Astronomy, \\ Johns Hopkins University, Baltimore, MD 21218, USA \\ ${ }^{b}$ Department of Physics, University of Washington, Seattle, WA 98195-1560, USA and \\ ${ }^{c}$ Department of Physics, Boston University, Boston, MA 02215
}

(Dated: October 22, 2018)

Cosmological inflation gives a natural answer for a variety of cosmological questions, including the horizon problem, the flatness problem, and others. However, inflation yields new questions relating to the flatness of the inflaton potential. Recent studies of "little" fields, a special class of pseudo-Goldstone bosons, have shown it is possible to protect the mass of a field while still yielding order one interactions with other fields. In this paper, we will show that "little inflatons" are natural candidates for the slow roll field of hybrid inflation models. We consider both supersymmetric and non-supersymmetric models, and give a simple examples based on approximate Abelian symmetries which solve the inflaton flatness problem of supergravity. We also present hybrid models in which components of gauge fields in higher dimensions play the role of the inflaton. Protected by higher-dimensional gauge symmetry, they, too, naturally have large couplings while suppressed mass terms. We summarize the implications of the new WMAP data on such models.

\footnotetext{
*dkaplan@pha.jhu.edu

† nealw@phys.washington.edu
} 


\section{INTRODUCTION}

Inflation [1] is an important feature of most modern cosmologies. It not only provides solutions for the horizon problem and the flatness problem, it also provides a natural explanation for the spectrum of density perturbations observed by COBE 2] and the more recent experiments such as MAXIMA [3], BOOMERANG [4] and DASI [5]. Even more recently, the WMAP [6, 7] experiment has added a great deal of new precision data, giving great new information into the physics of inflation.

In building models of inflation, one generally requires three basic ingredients: a large background energy density to drive inflation, a scalar field $\phi$ which will roll from some initial value to some other value where inflation ends, and the requirement that the energy associated with the kinetic energy is subdominant to the background energy density (the slow-roll conditions). With recent measurements, one can also constrain the form of the inflaton potential by studying density perturbations.

The simplest implementation of chaotic inflation [8] is just a scalar field with a polynomial potential. However, when the slow roll conditions (below) are applied, these models typically require super-Planckian vevs to drive inflation. In other words, because the potential is flat, one must start at very large field values to produce enough vacuum energy.

One interesting solution, hybrid inflation [9], represented significant progress in inflation model-building. If one field is responsible for slowly rolling while a different field is kept from relaxing to its minimum, then it is possible to divorce the size of the vacuum energy from the slow roll potential in a relatively natural way. In its original form, there are two fields, $\phi$, the slow-roll field and $\sigma$, the "waterfall" field, and a potential

$$
V(\phi, \sigma)=\lambda\left(M^{2}-\sigma^{2}\right)^{2}+\frac{m^{2}}{2} \phi^{2}+\frac{g^{2}}{2} \phi^{2} \sigma^{2}
$$

When the slow roll field has a large value, $\sigma$ has a large positive mass squared and is quickly driven to zero. From this point the universe inflates with an energy density $\sim \lambda M^{4}$ and $\phi$ slowly rolls until it reaches $\phi_{c} \equiv \sqrt{4 \lambda M^{2} / g^{2}}$ where the mass squared of $\sigma$ changes sign. At this point inflation ends.

The slow roll requirements are $\epsilon \ll 1$ and $|\eta| \ll 1$ where

$$
\epsilon \equiv \frac{M_{P l}^{2}}{2}\left(\frac{V^{\prime}}{V}\right)^{2}
$$




$$
\eta \equiv \frac{M_{P l}^{2} V^{\prime \prime}}{V} .
$$

These imply that $m^{2} \ll \lambda M^{4} / M_{P l}^{2}$ (where $M_{P l} \simeq 2 \times 10^{18} \mathrm{GeV}$, the reduced Planck mass). This introduces a significant model building problem: the (squared) mass of a scalar field receives quadratically divergent quantum corrections generically making it too large and making it sensitive to physics at the ultraviolet cutoff of the theory.

A technically natural mass for $\phi$, taking the cutoff to be $M_{P l}$, is $m^{2} \sim(g / 4 \pi)^{2} M_{P l}^{2}$. With an $m^{2}$ about a tenth this size (requiring $\sim 10 \%$ fine-tuning) it is possible to generate enough e-foldings of inflation

$$
\begin{aligned}
N\left(\phi_{i}\right) & =\frac{1}{M_{P l}^{2}} \int_{\phi_{c}}^{\phi_{i}} \frac{V}{V^{\prime}} d \phi \\
& =\int_{\phi_{c}}^{\phi_{i}} \frac{1}{\eta} \frac{d \phi}{\phi} \gtrsim 60
\end{aligned}
$$

and density perturbations consistent with the COBE data:

$$
V^{1 / 4}=6 \epsilon^{1 / 4} \times 10^{16} \mathrm{GeV} .
$$

However, the couplings in this model are $\lambda \sim 10^{-11}$ and $g^{2} \sim 10^{-10}$. In addition, the vevs are $M, \phi_{c} \sim M_{P l}$ thus requiring all higher-dimensional operators to be suppressed by similar factors. Such extreme values can be avoided at the cost of dramatically increasing the fine-tuning required.

There are only two known symmetries which can protect a scalar field from acquiring a mass: supersymmetry and the shift symmetry of a Goldstone boson. Supersymmetry would seem a natural choice because of the ubiquitous flat directions in supersymmetric theories, but inflation is driven by a vacuum energy density which breaks supersymmetry and generically gives Hubble-scale masses to all scalar fields in the theory [10].

The shift symmetry of a Nambu-Goldstone boson (NGB) will, indeed, protect it from acquiring a mass, even during inflation. However, the inflaton cannot be an exact NGB as this same shift symmetry would prohibit the coupling required in the hybrid model. Pseudo Nambu-Goldstone bosons (pNGB) have couplings which violate the shift symmetry by small amounts and thus these fields remain light. However, simply calling $\phi$ a pNGB is not enough to correct the above picture - the parameter $m^{2}$ still gets a one-loop quadratic divergent contribution from $\phi$ 's coupling to $\sigma$ which puts strain on the model. It is this contribution which must be suppressed. 
The first use of pNGBs for the inflaton was so-called "Natural Inflation" [11, 12] (see also [13]) in which a pNGB sat at a particular point in its potential from whence it could roll. They have also been used, for example, as the waterfall field in supersymmetric hybrid inflation models [14]. However, these models did not suitably take into account possible gravitational corrections, which can be significant. Indeed, in 14], the slow roll field would still naturally pick up a large mass from gravitational interactions.

Recently, a new class of pNGBs have been discovered [15] in which loop contributions to masses are suppressed. The suppression is due to the "non-local" or "collective" behavior of the explicit symmetry breaking: the breaking comes from a combination of couplings in the Lagrangian. The result is a vanishing tree-level mass and the cancelation of quadratic divergences at one or more loops. The first application of this mechanism was to the question of electroweak symmetry breaking [15], where the Higgs quartic arose from multiple symmetry breaking terms and the loop contributions to its mass were suppressed. A "Little Inflaton" theory is therefore one in which the slow-roll field $\phi$ is a little pNGB. It can have non-trivial interactions with the waterfall field (which itself may or may not be a pNGB) without generating large corrections to its mass.

We will show that the use of the above mechanism will maintain a flat potential for the slow-roll field against all dangerous corrections, including gravitational. In the next section we review the mechanism of collective symmetry breaking (CSB) and then present a little inflaton model.

In Section \س we review the difficulty in realizing inflation models in supersymmetric theories coupled to supergravity (the " $\eta$ problem"). We then present a simple supersymmetric model using the same mechanism as in the previous case. The dimensionless parameters are all order one and the model only requires one additional scale besides the Planck scale. We include a sequestered model in an extra dimension which gives a natural microscopic explanation for the form of the potential.

Another naturally light scalar with a protective shift symmetry is the fifth component of a gauge field in an extra dimension. In Section IV we present models of "gauge inflation" in which the slow roll field is the fifth component of a $U(1)$ gauge field, again achieving natural inflation with order one parameters. One can see from dimensional deconstruction [16] that these extra components of the gauge field are akin to pNGBs. We discuss the deconstruction of a particular six-dimensional model of this type. 
In Section $\square$ we discuss the significance of the recent WMAP results on the given models. Finally, in Section VI we conclude.

\section{GOLDSTONE BOSONS, COLLECTIVE BREAKING AND A NEW MODEL OF INFLATION}

Before discussing explicit models of inflation, we describe a new mechanism which leads to a naturally flat scalar potential. Until now, this mechanism has only been applied to electroweak symmetry breaking to produce a light Higgs boson mass compared to the cutoff of the theory. Here we apply it to the inflaton potential.

A NGB is a scalar field that results from a spontaneous broken global symmetry. For example, we can consider an $S O(2)$ symmetry broken by a linear sigma model field

$$
\Phi=e^{i \tau_{2} \theta\left(x^{\mu}\right) / f}\left(\begin{array}{c}
0 \\
f+\rho\left(x^{\mu}\right)
\end{array}\right)
$$

(where the normalization is chosen for notation ease later). It will frequently be convenient to set $\rho=0$, that is to consider the non-linear sigma model in which we do not discuss the dynamics of symmetry breaking. If $\Phi$ appears only in terms which respect the $S O(2)$ symmetry, the field $\theta$ will have only derivative interactions.

A pseudo- or approximate NGB is one in which the potential explicitly breaks the global symmetry with small couplings. An example of pseudo-NGBs in nature are the pions of QCD. Quark masses and electric charge break the chiral symmetry explicitly and give the pions masses at tree-level and one loop respectively.

The inflaton is a natural candidate for a NGB as we have discussed, because of the

necessary flatness of the potential. However, calling $\phi$ in the hybrid inflation model above a pNGB doesn't change the theory - specifically, $\phi$ still receives a large mass term via quantum effects.

The problem with the hybrid model considered as theory of a pNGB inflaton is that a single coupling in the potential broke the symmetry protecting $\phi$ from a mass term. A quadratically divergent renormalization was thus allowed to appear at one-loop, which is generically too large.

However, if the global symmetry is explicitly broken by a combination of couplings, then loop contributions to $p N G B$ masses must involve all of the couplings, and the one-loop 
contribution cannot be quadratically divergent. This is the essence of collective or non-local symmetry breaking [15]. The effect is to suppress loop contributions to the $\phi$ mass in the model of the previous section, naturally making the slow-roll parameter $\eta$ small.

\section{A. The littlest inflaton}

We begin by considering the simplest model of the type described above. It involves replacing $\phi$ with a pseudo-NGB $\theta$ which comes from the breaking of a global $S O(2)$ symmetry. We integrate out the "radial" degree of freedom $\rho$ and push the cutoff of this non-linear sigma model to the point where $\phi$ becomes strongly coupled, namely $\Lambda \sim 4 \pi f$. Thus the inflaton is parameterized as

$$
\Phi=\left(\begin{array}{cc}
\cos (\theta / f) & \sin (\theta / f) \\
-\sin (\theta / f) & \cos (\theta / f)
\end{array}\right)\left(\begin{array}{c}
-1 \\
1
\end{array}\right) \times \frac{f}{\sqrt{2}}
$$

We choose to parameterize the $S O(2)$ breaking in the direction above for convenience later. We take its tree-level potential to be:

$$
V=\lambda\left(\sigma^{T} \sigma-v^{2}\right)^{2}+\frac{g_{1}}{4}\left(\sigma^{T} \Phi\right)^{2}+\frac{g_{2}}{4}\left(\sigma^{T} \tau_{1} \Phi\right)^{2}
$$

where $\sigma^{T}=\left(\sigma_{1} \sigma_{2}\right)$ and $\tau_{1}$ is the first Pauli matrix.

When $g_{1}=g_{2}=0, \theta$ transforms non-linearly under an exact $S O(2)$ symmetry while $\sigma$ transforms linearly under a different $S O(2)$ symmetry. Making, for example, $g_{1}$ nonzero explicitly breaks the two symmetries to the diagonal $S O(2)$ which leaves $\left(\sigma_{1} \sigma_{2}\right) \cdot \Phi$ invariant. The remaining $S O(2)$ is still spontaneously broken and thus $\theta$ still represents an exact NGB. To see this, one can do a field redefinition which makes $\tilde{\sigma}^{T}=\sigma^{T} e^{-i \tau_{2} \theta / f}$, which turns the $g_{1}$ operator into a mass term for the linear combination $\left(\tilde{\sigma}_{1}-\tilde{\sigma}_{2}\right)$. The transformation changes the derivative operators in the theory (e.g., the kinetic terms for $\sigma$ produce higher-dimensional operators), but the potential is independent of $\theta$.

If instead we make $g_{2}$ non-zero, we still leave an $S O(2)$ symmetry preserved under which $\left(\sigma_{2} \sigma_{1}\right) \cdot \Phi$ is a singlet. We could again go to "unitary gauge" and transform $\theta$ away. However, with both terms present, we can only eliminate one term by a field transformation, leaving a non-trivial potential for $\theta$. However, since this potential and consequently the renormalization of the mass will require both $g_{1}, g_{2} \neq 0$, the slow-roll conditions are easily accommodated. 
For the rest of this discussion we take $g_{1}=g_{2} \equiv g$ for simplicity. This can be enforced by imposing a $\mathcal{Z}_{2}$ symmetry which takes $\sigma_{1} \leftrightarrow \sigma_{2}$. This simplification is not necessary for a successful model and the dynamics are not dramatically different in the more general case. In addition, we have imposed a $\sigma \rightarrow-\sigma$ symmetry on the Lagrangian to avoid terms linear in $\sigma$.

Now we compute the one-loop corrections to the mass of $\theta$. From expanding out the $\Phi$ s in the potential,

$$
V=\frac{g f^{2}}{4}\left(\sigma_{1}^{2}+\sigma_{2}^{2}-2 \sigma_{1} \sigma_{2} \cos (2 \theta / f)\right)
$$

it is clear that there is no one-loop quadratic divergent contribution to a $\theta$ mass. This is because $\theta$ only couples to the combination $\sigma_{1} \sigma_{2}$ making it impossible to close a loop with only one vertex. There is a logarithmic divergence at one loop proportional to $g_{1} g_{2}=g^{2}$

$$
\begin{aligned}
V_{1-\text { loop }} & =\frac{g^{2}}{128 \pi^{2}} \log \left(\frac{\Lambda^{2}}{m_{\theta}^{2}}\right)\left(\Phi^{T} \tau_{1} \Phi\right)^{2}+\ldots \\
& =\frac{g^{2} f^{4}}{128 \pi^{2}} \log \left(\frac{\Lambda^{2}}{m_{\theta}^{2}}\right) \cos ^{2}(2 \theta / f)+\ldots
\end{aligned}
$$

Because this theory is a non-linear sigma model, it becomes strongly coupled at a scale $\Lambda \simeq 4 \pi f$. We take this to be the cutoff of the theory but for now leave its value unspecified. It could be at $M_{P l}$ or far below.

Let us now compute the number of e-foldings. For simplicity, we take the VEV of the waterfall field $\sigma$ to be $v=f \simeq \Lambda / 4 \pi$. This value is not only technically natural, but the theory remains perturbative (and therefore under control) in this regime. To find the critical value of $\theta$, we diagonalize the $\sigma$ mass matrix:

$$
\begin{aligned}
& \sigma^{T} \frac{g}{4}\left(\begin{array}{cc}
1 & \cos (2 \theta / f) \\
\cos (2 \theta / f) & 1
\end{array}\right) \sigma \\
\rightarrow & \tilde{\sigma}^{T} \frac{g}{4}\left(\begin{array}{cc}
1-\cos (2 \theta / f) & 0 \\
0 & 1+\cos (2 \theta / f)
\end{array}\right) \tilde{\sigma}
\end{aligned}
$$

This gives us a value for $\theta_{c}$ satisfying

$$
\frac{4 \lambda}{g}=1-\cos \left(2 \theta_{c} / f\right)=2 \sin ^{2}\left(\frac{\theta_{c}}{f}\right) .
$$

The $\sigma$ field is stabilized when $|\theta|>\left|\theta_{c}\right|$. Note the sign of the mass term for $\theta$ needs to be opposite that which appears in the loop calculation. However, the sign is not a prediction 
of the theory as there is a counterterm canceling this logarithmic divergence at one loop (along with the two-loop quadratic divergence). We assume the sign is what we need for the proper dynamics while the magnitude is approximately that calculated above, i.e.,

$$
V_{\theta-m a s s} \simeq-\frac{g^{2} f^{4}}{128 \pi^{2}} \log \left(\frac{\Lambda^{2}}{m_{\theta}^{2}}\right) \cos ^{2}(2 \theta / f) \equiv-\frac{\bar{g}^{2}}{128 \pi^{2}} f^{4} \cos ^{2}(2 \theta / f),
$$

where $\bar{g}^{2}=g^{2} \log \left(\Lambda^{2} / m_{\theta}^{2}\right)$. The number of e-foldings can be computed

$$
\begin{aligned}
N\left(\theta_{i}\right) & =\frac{1}{M_{P l}^{2}} \int_{\theta_{c}}^{\theta_{i}} \frac{-128 \pi^{2} \lambda f^{4}}{2 \bar{g}^{2} f^{3} \sin (4 \theta / f)} d \theta \\
& =-\frac{\lambda}{\bar{g}^{2}} \log \left(\frac{\tan \left(2 \theta_{c} / f\right)}{\tan \left(2 \theta_{i} / f\right)}\right) \\
& \sim \frac{\lambda}{2 \bar{g}^{2}} \log (g / \lambda)
\end{aligned}
$$

where we have assumed $\theta_{c} \ll f$ and we've taken $\Lambda=M_{P l}=4 \pi f$. Thus we see from eq. (13) that $\lambda<g$ and from eq. (15) we require $\lambda \gg g^{2}$.

COBE requirements on the spectrum imply

$$
\frac{\lambda^{3 / 2}}{\pi \bar{g}^{2} \sin (4 \theta / f)}=5.2 \times 10^{-4}
$$

or, roughly,

$$
\bar{g}^{2} \approx \lambda^{3 / 2} \times 10^{3}
$$

Altogether, this implies $\bar{g}<10^{-3}$ and $\lambda<10^{-6}$. Numerically, then, we have $m_{\sigma}^{2} \approx 4 \lambda f^{2}<$ $\left(4 \times 10^{14} \mathrm{GeV}\right)^{2}$ and $m_{\theta}^{2} \approx \bar{g}^{2} f^{2} / 16 \pi^{2}<\left(2 \times 10^{13} \mathrm{GeV}\right)^{2}$.

Thus, as an illustration of the mechanism, we have shown an example of a hybrid model with only one additional coupling $\left(g \rightarrow g_{1}, g_{2}\right)$ which is technically natural, does not require cutoff-size vevs, and yet has a much smaller inflaton mass than would be otherwise expected.

\section{A SUPERSYMMETRIC LITTLE INFLATON}

Supersymmetry would seem a natural resolution to the flatness problem of the inflaton potential. After all, scalar fields in supersymmetric theories are protected by nonrenormalization theorems, and the abundant flat directions in supersymmetric theories seem natural candidates for the inflaton field.

However, since inflation necessarily involves gravity, it is necessary to embed the theory in supergravity. Because inflation occurs as a result of energy density, supersymmetry is 
broken during inflation and fields generically pick up masses of order the Hubble parameter, in direct conflict with slow-roll requirements [10].

Let us see explicitly how this happens: the potential for a scalar field during inflation is

$$
\begin{aligned}
V= & \exp \left(\frac{K}{M_{P l}^{2}}\right) \times \\
& {\left[\sum_{\alpha, \beta}\left(\frac{\partial^{2} K}{\partial \bar{\phi}_{\alpha} \partial \phi_{\beta}}\right)^{-1}\left(\frac{\partial W}{\partial \phi_{\alpha}}+\frac{W}{M_{P l}^{2}} \frac{\partial K}{\partial \phi_{\alpha}}\right)\left(\frac{\partial W}{\partial \bar{\phi}_{\beta}}+\frac{W}{M_{P l}^{2}} \frac{\partial K}{\partial \bar{\phi}_{\beta}}\right)-3 \frac{|W|^{2}}{M_{P l}^{2}}\right], }
\end{aligned}
$$

where $W$ is the superpotential and $K$ is the Kähler potential. Let us assume that the inflaton field has canonical Kähler potential, and that there is a source of vacuum energy, and thus supersymmetry breaking, in the $F$ term of some field, such that $F^{2}=\mu^{4}$. Then the inflaton field automatically picks up a mass through supergravity couplings,

$$
V=\exp \left(\frac{K}{M_{P l}^{2}}\right)\left(\mu^{4}+\ldots\right) .
$$

For a canonical Kähler potential $K\left(\phi^{*}, \phi\right)=\phi^{*} \phi$, this yields a slow-roll parameter $\eta=1$ at tree level, absent fine tunings of other terms in the potential.

There are at least two other approaches two address this: first of all, if the slow-roll field enters only linearly in the superpotential, there is a cancellation between this term and other terms [17]. However, this requires no additional Planck-scale vevs in the theory, such as dilaton vevs or moduli vevs - a highly restrictive assumption about the ultraviolet theory. Another alternative is D-term inflation [18], where the vacuum energy is driven by a Fayet-Iliopoulos term, although the scales in such models are often too high and it is difficult to avoid generating large $F$ terms.

\section{A. The Model}

As we have already described, a little pNGB is one which is a pseudo-goldstone boson, whose mass is protected by a combination of symmetries. In non-supersymmetric theories, this is typically realized with multiple approximate internal symmetries. In supersymmetric theories, masses are already protected by supersymmetry. For a pNGB to acquire a mass, only terms which combine both violations of supersymmetry as well as violations of the approximate internal symmetry can contribute.

A number of authors have previously considered models of pNGBs in supersymmetric theories. In [10], it was noted that pNGBs would not suffer from the supergravity $\eta$ problem. 
Models with discrete non-Abelian symmetries were constructed by [19]. In [20] such form of the Kähler potential was invoked, while [21] noted that moduli fields can naturally yield appropriate Kähler potentials. The authors of [22, 23] considered many models of pNGBs which have nearly exact symmetries except for small breaking parameters. In [14], pNGBs were considered as candidates for waterfall fields.

Goldstone bosons are protected by a shift symmetry, rather than supersymmetry. Hence, even in the presence of a cosmological constant, Goldstone bosons in supergravity are massless. Let us understand this more carefully. Suppose we have a field which spontaneously breaks a global $U(1)$ symmetry,

$$
W_{g b}=S\left(\Phi \bar{\Phi}-v^{2}\right)
$$

$F$-flatness is achieved when

$$
\begin{gathered}
\phi=v e^{\theta / \sqrt{2} v}, \\
\bar{\phi}=v e^{-\theta / \sqrt{2} v},
\end{gathered}
$$

where $\theta$ is a complex scalar field. We can promote $\theta$ to a superfield $\Theta$, in which case the kinetic terms are

$$
K\left(\Theta, \Theta^{\dagger}\right)=\Phi^{\dagger} \Phi+\bar{\Phi}^{\dagger} \bar{\Phi}=2 v^{2} \cosh \left(\frac{\Theta+\Theta^{\dagger}}{\sqrt{2} v}\right) .
$$

Let us then as before assume the presence of a field with a non-vanishing $F$-term. Then the the potential for $\theta$ in the presence of supergravity is

$$
V=\exp \left[\frac{2 v^{2}}{M_{P l}^{2}} \cosh \left(\frac{\theta+\theta^{*}}{\sqrt{2} v}\right)\right]\left(\mu^{4}+\ldots\right)
$$

Notice that since $K\left(\theta, \theta^{*}\right)$ depends only on the real component of $\theta$ and therefore only the real component acquires a mass! This is completely expected, because it is not supersymmetry protecting the imaginary component, but the shift symmetry of the goldstone boson. Indeed, any field $\Phi$ whose Kähler potential is a function of $\Phi+\Phi^{\dagger}$ alone has this same shift symmetry in the Kähler potential and will not acquire a mass. However, for an ordinary field, such a form for the Kähler potential would seem an unnatural tuning, whereas for a goldstone boson it is automatic.

We will consider a theory with a spontaneous breaking of an approximate little $U(1)$. All terms in the superpotential will respect a spontaneously broken $U(1)$, but in combination they will not. 
For the moment, we will not concern ourselves with the origin of this model, and will only address questions of technical naturalness. Later, we will comment on microscopic origins for the form given. The complete model is given by the superpotential

$$
W=X_{1}\left(\Phi \bar{\Phi}-v^{2}\right)+X_{2}\left(\frac{\kappa}{2} S^{2}-\mu^{2}\right)+\lambda S^{2}(\Phi+\bar{\Phi})
$$

The equality of the $\Phi$ and $\bar{\Phi}$ couplings can be justified by a $\Phi \leftrightarrow \bar{\Phi}$ exchange symmetry. Notice that each term respects a $U(1)$ under which $\Phi$ is charged, but in combination, there is no preserved $U(1)$. At a scale $v, \phi$ and $\bar{\phi}$ acquire vevs and we are left with a non-linear sigma model below this scale. We can thus instead study the theory

$$
W=X_{2}\left(\frac{\kappa}{2} S^{2}-\mu^{2}\right)+2 \lambda v S^{2} \cosh \left(\frac{\Theta}{\sqrt{2} v}\right)
$$

This model has a potential (neglecting supergravity contributions)

$$
V_{0}=\left|\frac{\kappa}{2} s^{2}-\mu^{2}\right|^{2}+\left|\kappa x_{2} s+4 \lambda v s \cosh \left(\frac{\theta}{\sqrt{2} v}\right)\right|^{2}+2 \lambda^{2}\left(s s^{*}\right)^{2} \frac{\left|\sinh \left(\frac{\theta}{\sqrt{2} v}\right)\right|^{2}}{\cosh \left(\frac{\theta+\theta^{*}}{\sqrt{2} v}\right)}
$$

Of course, because $\phi$ is just a pseudo-goldstone boson, radiative effects will give it a mass. There are two sources for such terms. The first is due to the fact that the form of the Kähler potential is unstable against radiative corrections, leading to induced $\phi^{\dagger} \bar{\phi}+$ h.c. Kähler potential terms. The contribution to the Kähler potential will be

$$
\delta K=\frac{\bar{\lambda}^{2}}{16 \pi^{2}}\left(\phi^{\dagger} \bar{\phi}+\text { h.c. }\right)
$$

where $\bar{\lambda}^{2}=\lambda^{2} \log (\Lambda / v)$. The $O\left(v^{2}\right)$ masses will drive $s$ to 0 , while supersymmetry breaking masses for $\theta_{r}$ will drive it to zero.

Another contribution to the potential for $\theta_{i}$ will come from the Coleman-Weinberg potential [24]. This is found to be

$$
\begin{array}{r}
\delta V=\frac{\kappa^{2} \mu^{4}}{128 \pi^{2}}\left[\left(x^{2}-1\right)^{2} \log \left(\left(x^{2}-1\right) \kappa \mu^{2} / \Lambda^{2}\right)+\right. \\
\left.\left(x^{2}+1\right)^{2} \log \left(\left(x^{2}+1\right) \kappa \mu^{2} / \Lambda^{2}\right)-2 x^{4} \log \left(x^{2} \kappa \mu^{2} / \Lambda^{2}\right)\right]
\end{array}
$$

where $x=4 \lambda v \cos \left(\theta_{i} / \sqrt{2} v\right) / \sqrt{\kappa} \mu$. Note that one cannot estimate which contribution is more relevant merely by which is larger because the slow roll conditions involve the derivatives of $\theta_{i}$ 's potential, not its absolute value. 
We will first consider each contribution in turn. If we assume the supergravity contribution is dominant, we have

$$
\begin{aligned}
V & =\exp \left(\frac{\bar{\lambda}^{2} v^{2}}{8 \pi^{2} M_{P l}^{2}} \cos \left(\frac{\sqrt{2} \theta_{i}}{v}\right)\right) \mu^{4} \\
& \simeq \mu^{4}+\frac{\bar{\lambda}^{2} v^{2} \mu^{4}}{8 \pi^{2} M_{P l}^{2}} \cos \left(\frac{\sqrt{2} \theta_{i}}{v}\right) .
\end{aligned}
$$

Note that this regardless of the particular value of $\theta_{i}$, the second term is a negligible contribution to the total energy. We can now calculate the slow roll parameters

$$
\begin{aligned}
& \epsilon=\frac{M_{P l}^{2}}{2}\left(\frac{V^{\prime}}{V}\right)^{2}=\frac{\bar{\lambda}^{4} v^{2}}{\left(8 \pi^{2}\right)^{2} M_{P l}^{2}} \sin ^{2}\left(\frac{\sqrt{2} \theta_{i}}{v}\right) \\
& \eta=\frac{M_{P l}^{2} V^{\prime \prime}}{V}=-\frac{\bar{\lambda}^{2}}{4 \pi^{2}} \cos \left(\frac{\sqrt{2} \theta_{i}}{v}\right),
\end{aligned}
$$

where we assume an arbitrary starting value for $\theta_{i} \sim v$. Because the potential for the slow roll field is generated at the loop level, both $\eta$ and $\epsilon$ are small. Indeed, $\epsilon$ is small even if $v \sim M_{P l}$.

The additional requirements on inflation are also easily satisfied. The total number of e-foldings during inflation is given by

$$
N=\int_{\phi_{i}}^{\phi_{f}} M_{P l}^{-2} \frac{\mu^{4}}{V^{\prime}} d \phi \simeq \frac{4 \pi^{2}}{\bar{\lambda}^{2}} \log \left(\frac{\tan \theta_{f}}{\tan \theta_{i}}\right) \simeq \frac{4 \pi^{2}}{\bar{\lambda}^{2}} \log \left(\frac{4 \lambda v}{\sqrt{\kappa} \mu}\right),
$$

requiring $\bar{\lambda}^{2} \sim O(1)$ to achieve 60 e-foldings, a very mild requirement. Note that this requirement combined with our expressions for the slow roll parameters tells us that we would expect a blue spectrum if the SUGRA terms dominate the inflation.

COBE constraints on density perturbations require the relationship between the scale of inflation and the symmetry breaking scale

$$
\mu^{2}=10^{-5}\left(\bar{\lambda}^{2}\right) v M_{P l}
$$

Since we require $v>\mu$ in order to achieve a workable hybrid inflation model, this implies

$$
v \geq \times 10^{-5}\left(\bar{\lambda}^{2}\right) M_{P l} \approx\left(\bar{\lambda}^{2}\right) 2 \times 10^{13} \mathrm{GeV},
$$

where this limit is saturated in the limit that $v=\mu$. Note that in the other limit, $v=M_{P l}$ we derive

$$
\mu \approx \overline{8} \lambda \times 10^{15} \mathrm{GeV}
$$


Now let us assume the Coleman-Weinberg contribution is dominant. Then we have slow roll parameters

$$
\begin{aligned}
& \epsilon \simeq \frac{M_{P l}^{2} \kappa^{4} \tan ^{2}\left(\sqrt{2} \theta_{i} / v\right)}{1024 \pi^{4} v^{2}} \\
& \eta \simeq-\frac{M_{P l}^{2} \kappa^{2} \sec ^{2}\left(\sqrt{2} \theta_{i} / v\right)}{14 \pi^{2} v^{2}}
\end{aligned}
$$

We thus have $\epsilon / \eta=\kappa^{2} \sin ^{2}(\theta / \sqrt{2} v) / 64 \pi^{2}$, so $|\eta|>\epsilon$. Consequently, we would expect a red spectrum if the CW potential dominates the dynamics of inflation.

The number of efoldings is

$$
N \approx \frac{16 \pi^{2} v^{2}}{M_{P l}^{2} \kappa^{2}} \log \left(x_{i}\right),
$$

hence for large $v$, order one $\kappa$ will suffice, while in general we require $\kappa \sim v / M_{P l}$ to achieve the necessary inflation. COBE requires

$$
\mu^{2} v \simeq 2.3 \times 10^{-6} M_{P l}^{3} \kappa^{2} \tan \left(\sqrt{2} \theta_{i} / v\right)
$$

In conclusion, in both scenarios (supergravity dominated and Coleman-Weinberg dominated) the loop factor is responsible for both the slow roll and the large number of e-foldings. The density perturbations make a requirement on the scales in the theory. However, while $\mu \ll v \ll M_{P l}$ is consistent with the theory, we do not require multiple hierarchies in the theory. Both $\mu \sim v$ and $v \sim M_{P l}$ are consistent scenarios with only two scales present and no other very small parameters, and no other small parameters at all if $v \sim M_{P l}$.

\section{B. Sequestered Realizations}

The theory presented is technically natural, in the sense that some couplings are included only at their radiatively induced size. It is interesting to consider whether there are microscopic realizations which set these couplings to zero at tree level automatically. Here we will discuss the use of sequestering to achieve this, while in section IV we will consider models where the inflaton is part of a higher dimensional gauge field.

While the precise model described above is difficult to realize on the basis of symmetries and sequestering, a very similar model can be. Let us consider a five dimensional theory on $S_{1} / Z_{2}$ with a gauged $U(1)$ propagating in the fifth dimension. Let us further suppose that we spontaneously break the $U(1)$ on two boundaries by fields $\Phi_{1,2}, \bar{\Phi}_{1 / 2}$ with charges 
\pm 1 , and that a bulk hypermultiplet $S, \bar{S}$ propagates in the fifth dimension and has charge $\pm 1 / 2$ under the $U(1)$. Then we can go to the gauge where $\Phi_{1}=\bar{\Phi}_{2}=\Phi=e^{\theta / 2 v}$ and $\bar{\Phi}_{1}=\Phi_{2}=\bar{\Phi}=e^{-\theta / 2 v}$.

The superpotential is constrained because no direct couplings between $\phi_{1}$ and $\phi_{2}$ can occur due to locality. However, we can write

$$
W \supset X\left(S \bar{S}-\mu^{2}\right)+\bar{S}^{2}\left(\Phi_{1}+\Phi_{2}\right)+S^{2}\left(\bar{\Phi}_{1}+\bar{\Phi}_{2}\right)
$$

which, going to the nonlinear sigma model yields

$$
W \supset X\left(S \bar{S}-\mu^{2}\right)+\bar{S}^{2}(\Phi+\bar{\Phi})+S^{2}(\bar{\Phi}+\Phi)
$$

This is a minor modification of the earlier model, and the subsequent analysis follows with only trivial changes.

\section{INFLATION FROM EXTRA DIMENSIONS}

Now we present models of the inflaton as a component of a higher-dimensional gauge field. In a five-dimensional theory with a $U(1)$ gauge symmetry, $A_{5}$, the fifth component of the gauge boson, is protected by a remnant of the full gauge symmetry and therefore its mass is cut off by the compactification scale, making it a perfect candidate for an inflaton. It is the Wilson line which is simply a pNGB as is clearly shown by deconstruction [16]. Finally, we present a supersymmetric version of gauge inflation which is to some extent a "reconstructed" version of the model in section III. We briefly describe a six-dimensional version with $A_{6}$ as the waterfall field, and its deconstruction. Adding compact extra dimensions requires their stabilization, which we shall assume happens independent of these models. This assumption is of course not required in the deconstructed case. Note that components of gauge fields in extra (and deconstructed) dimensions has been suggested as a mechanism for quintessence 25]. 


\section{A. Gauge Inflation}

The Lagrangian of a $U(1)$ gauge theory in five dimensions compactified on a circle, with a charged scalar $\sigma$ in the bulk is

$$
\mathcal{L}_{5}=\left|\partial_{M} \sigma+i g_{5} A_{M} \sigma\right|^{2}+\frac{1}{2} F^{M N} F_{M N}+V\left(|\sigma|^{2}\right)
$$

where $M, N=0,1,2,3,5$ and the Lagrangian has dimensions of (mass) $)^{5}$. The field $A_{5}(x, y)$ will have an initial non-zero value before the 60 e-foldings. Five-dimensional gauge invariance allows one to transform a general profile for the field in the fifth direction to one which is flat (independent of $y$. This is the Wilson line which, below the compactification scale $M_{c} \equiv R^{-1}=(2 \pi L)^{-1}$, is simply a scalar field. This is the scale at which loop corrections to the zero-mode $\left(A_{5}^{0}\right)$ mass are cut off. This motivates us to consider $A_{5} \rightarrow \phi$ as a slow roll field.

Let us begin by considering the simplest theory with one extra dimension and a charged scalar field. We will take the fundamental scale $M_{*}$ to be a free parameter, up to the requirement that $M_{*}>L^{-1}$. If the one extra dimension is the only parametrically large dimension, then we have the relation $M_{P l}^{2}=M_{*}^{3} L$ [26]. However, if there are other extra dimensions in which the fields we consider do not propagate, the fundamental scale can be lower.

The potential we will consider will be

$$
V=\lambda\left(|\sigma|^{2}-v^{2}\right)^{2}+g^{2}|\sigma|^{2} \phi^{2}
$$

Radiative effects tell us that the natural size are $\lambda \gtrsim g^{4} / 16 \pi^{2}$ and $v^{2} \sim M_{*}^{2} / 16 \pi^{2}$ if $\lambda>g^{2}$ and $g^{2} M_{*}^{2} / \lambda 16 \pi^{2}$ if $\lambda<g^{2}$. Note that we have not yet included any potential for $\phi$.

The potential for $\phi$ is a subtle issue. Because $\phi$ is identified with the fifth component of a gauge field in an extra dimension, the dynamics which generates it arises only from non-local interactions. Specifically, it will arise from loops of charged fields which propagate all the way around the extra dimension. However, these loops will be exponentially suppressed if the masses are large. For $\sigma$, which have a negative mass-squared, in order to have a $\sigma=0$ stable during inflation, we will need to take $\left|m_{\sigma}^{2}\right|<1 / g L$.

One can calculate the effective potential exactly, as the Lagrangian is quadratic in $\sigma$.

$$
\delta V=\int \frac{d^{4} q}{(2 \pi)^{4}} \sum_{n=-\infty}^{\infty} \log \left[(n+g \phi R)^{2}+(q R)^{2}+(m R)^{2}\right]
$$




$$
=\int \frac{d^{4} q}{(2 \pi)^{4}} \log \left(\frac{\sin (i f \pi-g \phi \pi R) \sin (i f \pi+g \phi \pi R)}{\sin ^{2}(i \pi f)}\right),
$$

where $f=R \sqrt{m^{2}+q^{2}}$.

When the waterfall field has a small mass compared to the compactification scale, this is well approximated by a cosine potential of the form

$$
\delta V \approx A(1-\cos (2 g \pi R \phi))
$$

where

$$
A=\frac{93 \zeta(5)}{1024 R^{4} \pi^{6}} \simeq \frac{3}{32 R^{4} \pi^{6}}
$$

The slow roll parameters are given by

$$
\begin{aligned}
\epsilon & =\frac{9 g^{2} M_{P l}^{2}}{512 \pi^{10} \lambda^{2} v^{8} R^{6}} \sin ^{2}(2 g \pi R \phi) \\
\eta & =\frac{3 g^{2} M_{P l}^{2}}{8 \lambda v^{4} \pi^{4} R^{2}} \cos (2 g \pi R \phi) .
\end{aligned}
$$

The total efoldings are

$$
N=\frac{8 \lambda \pi^{4} R^{2} v^{4}}{3 g^{2} M_{P l}^{2}} \log \left(\frac{\tan \left(g \pi R \theta_{i}\right)}{\tan \left(g \pi R \theta_{f}\right)}\right) .
$$

There are two interesting limits we can take, one in which the vev of $\sigma$ is as small as possible and one in which it is as large as possible $\left(\lambda v^{4} \sim 1 /(R)^{4}\right)$. We can reexpress $M_{P l}^{2}=M_{*}^{3} 2 \pi R V_{n}$, where $V_{n}$ is the additional volume in fundamental units.

In the small vev case we have

$$
\begin{aligned}
\epsilon & =\frac{2304 \sin ^{2}(2 g \pi R \phi) V_{n}}{g^{2} M_{*}^{5} R^{5} \pi x^{4}}, \\
\eta & =\frac{192 \pi \cos (2 g \pi R \phi) V_{n}}{M_{*} R x^{2}}
\end{aligned}
$$

where $x=g / \sqrt{\lambda}(\sqrt{\lambda} / g)$ if $g^{2}>\lambda\left(\lambda>g^{2}\right)$. If we take a large energy density (of order the compactification scale) we have

$$
\begin{aligned}
\epsilon & =\frac{9 g^{2}\left(M_{*} R\right)^{3} \sin ^{2}(2 g \pi R \phi) V_{n}}{256 \pi^{9}}, \\
\eta & =\frac{3 g^{2} \pi\left(M_{*} R\right)^{3} \cos (2 g \pi R \phi) V_{n}}{4 \pi^{3}} .
\end{aligned}
$$

Irrespective of the other parameters of the model, we have from COBE

$$
\eta \simeq 83 \cos (2 g \pi R \phi)\left(\frac{g^{4}}{\sin ^{2}(2 g \pi R \phi)}\right)^{1 / 3}
$$

so we need a somewhat small $g$ in order to satisfy density pertubations. 


\section{B. Pseudo-pseudo: the waterfall field}

It is possible to make both the slow-roll and waterfall fields components of gauge fields and thus have a theory without fundamental scalars. By going to six dimensions, the job of the waterfall field can be done by $A_{6}$. We will take a compactification scale for the sixth dimension higher than that for the fifth dimension, i.e., $L_{6}^{-1}>L_{5}^{-1}$.

Start with a six-dimensional $S U(2)$ gauge theory compactified with boundary conditions along the sixth dimension which break the $S U(2)$ to $U(1)$. This can be done by an orbifold such that under the transformation $x_{6} \rightarrow-x_{6}$, the $A_{\mu}^{0}, A_{5}^{0}, A_{6}^{ \pm}$are even and the $A_{\mu}^{ \pm}, A_{5}^{ \pm}, A_{6}^{0}$ are odd. Only the even fields have zero mode and so below the scale $L_{6}^{-1}$, there is a fivedimensional $U(1)$ gauge field and a charged scalar which we identify as $\sigma \equiv A_{6}^{+} / \sqrt{L_{6}}$.

The necessary coupling between inflaton and waterfall appears automatically as a result of gauge invariance. The gauge kinetic term contains $F_{56} F^{56}$ which in turn contains terms $\operatorname{Tr}\left[A_{5}^{0}, \sigma^{ \pm}\right]^{2}$. In addition, a potential for $\sigma$ is generated below $L_{6}^{-1}$. Thus the inflatonwaterfall coupling is built in! All that is needed is a potential for the waterfall field. This can be generated by a fermion doublet living on a boundary of the fifth dimension (say at $x_{5}=0$ ), or living in the full bulk with a mass $m_{f} \sim L_{6}^{-1} \gg L_{5}^{-1}$. The fermion will couple to $A_{6}$ through normal gauge interactions and give a negative squared mass and positive quartic term to the waterfall field, similar to the top contributions to the Higgs effective potential in the standard model of particle physics. Below the compactification scale of the sixth dimension, the dynamics are as in the five-dimensional model of the previous section.

We can deconstruct the six-dimensional model above, i.e., latticize the two extra dimensions. However, we can retain the salient features of the model above with very few lattice sites: two to be precise.

The model is of the same form as the "minimal moose" 27] model used to explain a light Higgs and electroweak symmetry breaking. There are four sets of link fields:

$$
\Phi_{i}=f e^{i \tilde{\theta}_{i} / f}=f e^{i \theta^{a} \tau^{a} / f}
$$

which each parameterize the breaking of $S U(2)_{L} \times S U(2)_{R} \rightarrow S U(2)_{V}$, just like the pions of QCD. Two $U(1)$ symmetries are gauged, $U(1)_{L} \times U(1)_{R}$, such that all links transform as

$$
\Phi_{i} \rightarrow e^{-i \tau^{3} \alpha(x)_{L}} \Phi_{i} e^{i \tau^{3} \alpha(x)_{R}} .
$$


In other words, we have gauged a $U(1)$ subgroup of the four $S U(2)_{L} \mathrm{~S}$ and similarly for the $S U(2)_{R}$. This is the equivalent to orbifolding. Adding the operators

$$
g_{1} \operatorname{Tr} \Phi_{1} \Phi_{2}^{\dagger} \Phi_{3} \Phi_{4}^{\dagger}+g_{2} \operatorname{Tr} \Phi_{1} \Phi_{4}^{\dagger} \Phi_{3} \Phi_{2}^{\dagger}
$$

produces a quartic potential mixing the neutral and charged components of the $\Phi \mathrm{s}$. Both couplings are required to produce a quartic-type coupling at tree level, and both are required to break the symmetries of some of the fields, thus suppressing one-loop contributions to their masses. Using the $U(1)$ gauge couplings as spurions, it is possible to introduce or generate a suitable potential for all of the fields, allowing for technical naturalness to suppress the sizes of operators.

While we do not explore the full details of this model here, we note that it would be of interest to produce a "moose" model [28] from a theory of fermions and gauge fields and without fundamental scalars.

\section{Supersymmetry in Extra Dimensions}

These extensions to higher dimensional gauge theories also work well in the supersymmetric models. Again, let us consider a five-dimensional $U(1)$ gauge theory with a bulk hypermultiplet $S, \bar{S}$. The action for the bulk fields [29] (including the radion [30]) are (we follow the notation of [31])

$$
\begin{aligned}
S_{5} & =\int d^{4} x d \phi\left[\int d^{2} \theta \frac{T}{4 g_{5}^{2}} W^{\alpha} W_{\alpha}+\bar{S}\left(\partial_{\phi}-\frac{\chi}{\sqrt{2}}\right) S+\text { h.c. }\right] \\
& +\int d^{4} \theta \frac{1}{T+T^{\dagger}}\left[\frac{2}{g_{5}^{2}}\left(\partial_{\phi} V-\frac{1}{\sqrt{2}}\left(\chi+\chi^{\dagger}\right)\right)^{2}\right]+\frac{T+T^{\dagger}}{2}\left(S^{\dagger} S+\bar{S}^{\dagger} \bar{S}\right) .
\end{aligned}
$$

We then add a boundary term which explicitly breaks the $N=2$ supersymmetry and the $U(1)$

$$
S_{4}=\int d^{4} x d \phi \delta(\phi) \int d^{2} \theta X\left(\frac{\kappa}{2} S \bar{S}-\mu^{2}\right) .
$$

Notice that once the radion is stabilized to a value $R$, this reduces to a a field with the appropriate Yukawa superpotential term, and most importantly, a kinetic term that depends only on $\chi+\chi^{\dagger}$. This is a remnant of the shift symmetry of the theory which protects the field from getting a mass. Of course, below the compactification, there is no remnant of the higher dimensional gauge theory and radiative effects should spoil this as in the little inflaton model. 
The fact that an $N=2$ gauge theory coupled to charged matter has the proper form for a hybrid inflation model has been noted by [32] and 33] and later studied by [34]. These models were not little inflaton models, however, because the Kähler potential employed was minimal, and they did not arise from fundamentally higher dimensional gauge theories. There the supergravity $\eta$ problem was solved by assuming the absence of Planck scale vevs in 32] and was solved by driving inflation with Fayet-Iliopoulos terms D-terms in [33]. F-term inflation would still have induced a large mass in this latter case.

In our model, the particular form of the Kähler potential is enforced by the five dimensional gauge symmetry, but below the compactification scale, radiative effects will spoil this. Moreover, contributions from the Coleman-Weinberg potential will also give a mass to $\chi$.

The corrections to the Kähler potential will be

$$
\delta K=-\frac{g^{2}}{16 \pi^{2}} \log (\langle\chi\rangle L) \chi \chi^{\dagger},
$$

while the Coleman-Weinberg contributions will be

$$
\begin{aligned}
\delta V= & \frac{\kappa^{2} \mu^{4}}{64 \pi^{2}}\left[-2 x^{4} \log \left(\frac{x^{2} \kappa \mu^{2}}{\Lambda^{2}}\right)+\left(x^{2}-1\right)^{2} \log \left(\frac{\left(x^{2}-1\right) \kappa \mu^{2}}{\Lambda^{2}}\right)\right. \\
& \left.+\left(x^{2}+1\right)^{2} \log \left(\frac{\left(x^{2}+1\right) \kappa \mu^{2}}{\Lambda^{2}}\right)\right]
\end{aligned}
$$

where $x=g \chi / \sqrt{2 \kappa} \mu$. This yields for $x \gg 1$

$$
\delta V \approx \frac{\kappa^{2} \mu^{4}}{32 \pi^{2}} \log \left(x^{2} \kappa \mu^{2} / \Lambda^{2}\right) .
$$

Again we consider each case in turn, first when supergravity corrections are dominant and then when Coleman-Weinberg contributions are dominant.

We begin with supergravity contributions, which give a mass term

$$
\delta V \approx-\frac{g^{2} \mu^{4}}{16 \pi^{2} M_{P l}^{2}} \log (\langle\chi\rangle L) \chi \chi^{*} .
$$

The slow roll parameters are then

$$
\begin{array}{r}
\epsilon=\frac{g^{4}}{128 \pi^{4} M_{P l}^{2}} \chi \log (\langle\chi\rangle L)^{2}, \\
\eta=-\frac{g^{2}}{8 \pi^{2}} 2 \log (\chi L) .
\end{array}
$$

Thus, slow roll is easily achieved, even with $O(1) \mathrm{g}$. The number of efoldings is

$$
N=\frac{8 \pi^{2}}{g^{2}} \log \left(\log \left(\chi_{i} L\right) / \log \left(\chi_{f} L\right)\right),
$$


while COBE requires

$$
\mu \approx 6 \times 10^{16} \mathrm{GeV} \frac{g}{2 \pi} \sqrt{\chi \log (\pi / L \chi) / 2 \sqrt{2} M_{P l}} .
$$

If the Coleman-Weinberg potential drives the slow-roll field, this essentially the model of 35], and we have slow roll parameters

$$
\begin{aligned}
& \epsilon=\frac{g^{2} \kappa^{3} M_{P l}^{2} x^{2}}{4(4 \pi)^{4} \mu^{2}}\left(x^{2} \log \left(\frac{x^{4}}{x^{4}-1}\right)+\log \left(\frac{x^{2}-1}{x^{2}+1}\right)\right)^{2}, \\
& \eta=-\frac{g^{2} \kappa M_{P l}^{2}}{32 \pi^{2} \mu^{2}}\left(3 x^{2} \log \left(\frac{x^{4}}{x^{4}-1}\right)+\log \left(\frac{x^{2}-1}{x^{2}+1}\right)\right) .
\end{aligned}
$$

For $x \gg 1$ these simplify to

$$
\begin{aligned}
\epsilon & =\frac{g^{2} \kappa^{3} M_{P l}^{2}}{4(4 \pi)^{4} \mu^{2} x^{2}}, \\
\eta & =-\frac{g^{2} \kappa M_{P l}^{2}}{32 \pi^{2} \mu^{2} x^{2}} .
\end{aligned}
$$

Note that we have $\epsilon=\kappa^{2} \eta / 32 \pi^{2}$. So merely by perturbativity $(\kappa<4 \pi)$ we have a $|\eta|>\epsilon$. The number of efoldings is

$$
N=\frac{16 \pi^{2} \mu^{2} x_{i}^{2}}{g^{2} \kappa M_{P l}^{2}},
$$

so even for $O(1) \kappa, g$, we can have sufficient inflation. Given the relatively simple form for $N$, we can rewrite the slow roll parameters at the COBE era as

$$
\begin{array}{r}
\epsilon \simeq \frac{\kappa^{2}}{64 \pi^{2} N_{C O B E}}, \\
\eta \simeq \frac{-1}{2 N_{C O B E}} .
\end{array}
$$

Lastly COBE requires

$$
\mu=6 \times 10^{16} \mathrm{GeV} \sqrt{\frac{k}{8 \pi \sqrt{N_{C O B E}}}} .
$$

In conclusion, the corrections to the potential are sufficiently soft that slow roll is easily achieved, along with satisfying COBE requirements, all without any very large or small parameters.

\section{IMPLICATIONS OF WMAP}

With the release of the new WMAP data [6, 7], cosmology has moved into a new era of precision data. In particular, we have a great deal of information now on the tilt parameter $n$, 
and tantalizing hints for possible non-zero $d n / d \log k$. A broad discussion of the implications for inflation is given in [36].

Let us begin by simply summarizing the WMAP results: from WMAP alone, $n=0.99 \pm$ 0.04, while inclusion of 2dFGRS [37, 38] and studies of Lyman $\alpha$ systems [39, 40] gives $n=0.93 \pm 0.03$. From this we see the data prefer red spectra, but are still consistent with blue spectra. These results assume a running spectral index, which is found to have $d n / d \log (k)=-0.031_{-0.017}^{+0.016}$. This suggests that $n$ is moving red on small distance scales, and a particularly remarkable indiciation is that the spectrum actually moves from blue $(n>1)$ on long distance scales to red $(n<1)$ on short distance scales. In light of this, we will examing the models studied here, beginning with the non-supersymmetric models and moving on to the supersymmetric ones.

The "littlest inflaton" model of section II has very small couplings, which are a priori independent. Without any explanation of their origin, one requires a fine-tuning of parameters in order to achieve any observable deviation from $n=1$. However, if such a tuning did occur, one would require the log enhancement of equation 15 (that is, $\tan \left(2 \theta_{i} / f\right) \gg 1$ ), and hence $\eta<1$ would be possible, allowing a red spectrum.

Next we consider the non-supersymmetric "gauge inflation" model. Here we have the relation

$$
\epsilon \approx \frac{3}{64 \lambda \pi^{6} R^{4} v^{4}} \eta
$$

Hence, if the inflation scale $\left(\lambda v^{4}\right)$ is small compared to the compactification scale, it is natural to have $\epsilon>|\eta|$, yielding a red spectrum. However, if $|\eta|>\epsilon$, because the potential involves a cosine, $\eta$ can have either sign. As a consequence it is difficult to make predictions with this model. However, $d n / d \log k$ can only be negative if $\epsilon$ is significant (yielding possibly observable tensor perturbations and a red spectrum) or in the top half of the potential (where the curvature is negative). Hence, if the observations of a spectrum changing from blue to red are verified, this non-supersymmetric model will be excluded.

The supersymmetric models are perhaps the most interesting because they have two sources to their potential: one from the Coleman-Weinberg potential and one from supergravity corrections. This is analagous to the situation in [17], with two important differences: first, we have no need to make additional assumptions about the short-distance physics of the theory. Unlike [17], we do not need to assume small vevs for all moduli fields, and all dangerous higher dimension operators which could give a large mass to the slow roll field 
are absent at tree level. This arises from the shift symmetry of the goldstone boson or the five-dimensional gauge symmetry. Secondly, the theory is entirely predictive from the renormalizable parameters of the theory - the non-renormalizable operators which are significant are those that arise out of the tree-level supergravity potential.

This being said, let us study the supersymmetric models in more detail. We will focus on the extra dimensional model, while the deconstructed model is quite similar with $1 / L$ identified with $v$. Recall that in the $\mathrm{CW}$ regime, $\eta \approx-1 / 2 N$. The transition between the CW and the SUGRA regime (when $V_{S U G R A}^{\prime}=V_{C W}^{\prime}$ and $V_{S U G R A}^{\prime \prime}=V_{C W}^{\prime \prime}$ ) occurs at $\chi=M_{P l} \kappa / \sqrt{2} g$ (here we ignore the logarithmic dependence of the SUGRA potential, keeping only the dominant power behavior and setting $\bar{g} \sim g$ ). The efoldings in the CW regime is then

$$
N_{C W}=\frac{4 \pi^{2}}{g^{2}}
$$

Hence, for order one $g$, the transition from SUGRA to CW naturally occurs at $O(40)$ efoldings before the end of inflation. As pointed out in [17], this is interesting because the transition from SUGRA to CW regimes gives a transition from a blue to a red spectrum, which is a feature suggested at $2 \sigma$ from the WMAP data. However, unlike 17] this is quite natural with order one parameters, and does not require additional small parameters.

In summary, the analyzed models are consistent with the WMAP data, although the "littlest inflaton" would appear finely tuned if there is increasing certainty of $n \neq 1$. The nonsupersymmetric gauge inflation model can satisfy a red spectrum with non-zero $d n / d \log k$, but cannot accomodate a change from blue to red. If the data continue to support this, this model will be excluded. The supersymmetric models quite naturally give all the qualitative features observed. A change from blue to red is quite natural in the regime suggested by WMAP, and, in fact arises in the model without any fine tuning of the parameters, or any assumptions about the coefficients of non-renormalizable operators. A complete study of these models is therefore warranted.

\section{DISCUSSION}

Inflation solves the big problem of the flatness of the universe, leaving a little problem in its wake - the flatness of the inflaton potential. This new class of particles - pNGBs with collective symmetry breaking - fits perfectly in this role. 
It is clear now that fundamental scalars are not required for inflation. Higher components of extra-dimensional gauge fields can contain all of the required dynamics. Little inflaton models can be generated by confining theories of fermions and gauge fields, possibly even to construct renormalizable ones (i.e., no n-fermion operators required).

For pNGBs in supersymmetric theories, the $\eta$-problem is solved. The kinetic term for these fields always come in the form $T+T^{\dagger}$, and any modulus could potentially play the role of the inflaton. For example, if $T$ were the radion multiplet in a five-dimensional supersymmetric theory, its imaginary scalar component is the fifth component of the graviphoton $B_{5}$. Thus it may be that a model which stabilizes the compact dimension already has all of the ingredients to produce inflation.

Previous discussions of pNGBs have involved either axion-like potentials (so-called "natural inflation") or situations where the approximate symmetry was very good, in that the explicit symmetry breaking parameters were very small. Here, we need not rely on extremely high scales (as in natural inflation) or very small parameters. Moreover, because the dynamics is more complicated, there is a greater hope for observable consequences in the CMBR.

Acknowledgments The authors would like to thank Andy Cohen for very useful conversations on inflation and Nemanja Kaloper for reading the draft and useful comments, as well as Ann Nelson and Raman Sundrum for useful discussions. The authors would also like to thank the theory group at Boston University where much of this work was undertaken.

Note added: As this work was being completed, we became aware of [41], which also considers the $A_{5}$ as a candidate for the inflaton, as well as work in progress by the same authors on similar ideas.

[1] A. H. Guth, Phys. Rev. D23, 347 (1981).

[2] C. L. Bennett et al., Astrophys. J. 464, L1 (1996), astro-ph/9601067.

[3] A. T. Lee et al., Astrophys. J. 561, L1 (2001), astro-ph/0104459.

[4] P. de Bernardis et al., Astrophys. J. 564, 559 (2002), astro-ph/0105296.

[5] J. Kovac, E. M. Leitch, C. P. J. E. Carlstrom, and N. W. H. W. L. Holzapfel (2002), astro- 
$\mathrm{ph} / 0209478$.

[6] C. L. Bennett et al. (2003), astro-ph/0302207.

[7] D. N. Spergel et al. (2003), astro-ph/0302209.

[8] A. D. Linde, Phys. Lett. B129, 177 (1983).

[9] A. D. Linde, Phys. Rev. D49, 748 (1994), astro-ph/9307002.

[10] E. J. Copeland, A. R. Liddle, D. H. Lyth, E. D. Stewart, and D. Wands, Phys. Rev. D49, 6410 (1994), astro-ph/9401011.

[11] K. Freese, J. A. Frieman, and A. V. Olinto, Phys. Rev. Lett. 65, 3233 (1990).

[12] F. C. Adams, J. R. Bond, K. Freese, J. A. Frieman, and A. V. Olinto, Phys. Rev. D47, 426 (1993), hep-ph/9207245.

[13] J. A. Frieman, C. T. Hill, A. Stebbins, and I. Waga, Phys. Rev. Lett. 75, 2077 (1995), astro$\mathrm{ph} / 9505060$.

[14] L. Randall, M. Soljacic, and A. H. Guth, Nucl. Phys. B472, 377 (1996), hep-ph/9512439.

[15] N. Arkani-Hamed, A. G. Cohen, and H. Georgi, Phys. Lett. B513, 232 (2001), hepph/0105239.

[16] N. Arkani-Hamed, A. G. Cohen, and H. Georgi, Phys. Rev. Lett. 86, 4757 (2001), hepth/0104005.

[17] A. D. Linde and A. Riotto, Phys. Rev. D56, 1841 (1997), hep-ph/9703209.

[18] P. Binetruy and G. R. Dvali, Phys. Lett. B388, 241 (1996), hep-ph/9606342.

[19] J. D. Cohn and E. D. Stewart, Phys. Lett. B475, 231 (2000), hep-ph/0001333.

[20] H. Murayama, H. Suzuki, T. Yanagida, and J. Yokoyama, Phys. Rev. D50, 2356 (1994), hep-ph/9311326.

[21] J. A. Adams, G. G. Ross, and S. Sarkar, Phys. Lett. B391, 271 (1997), hep-ph/9608336.

[22] M. Kawasaki, M. Yamaguchi, and T. Yanagida, Phys. Rev. Lett. 85, 3572 (2000), hep$\mathrm{ph} / 0004243$.

[23] M. Yamaguchi, Phys. Rev. D64, 063502 (2001), hep-ph/0103045.

[24] S. R. Coleman and E. Weinberg, Phys. Rev. D7, 1888 (1973).

[25] C. T. Hill and A. K. Leibovich, Phys. Rev. D66, 075010 (2002), hep-ph/0205237.

[26] N. Arkani-Hamed, S. Dimopoulos, and G. R. Dvali, Phys. Lett. B429, 263 (1998), hep$\mathrm{ph} / 9803315$.

[27] N. Arkani-Hamed et al., JHEP 08, 021 (2002), hep-ph/0206020. 
[28] H. Georgi, Nucl. Phys. B266, 274 (1986).

[29] N. Arkani-Hamed, T. Gregoire, and J. Wacker, JHEP 03, 055 (2002), hep-th/0101233.

[30] D. Marti and A. Pomarol, Phys. Rev. D64, 105025 (2001), hep-th/0106256.

[31] D. E. Kaplan and N. Weiner (2001), hep-ph/0108001.

[32] T. Watari and T. Yanagida, Phys. Lett. B499, 297 (2001), hep-ph/0011389.

[33] R. Kallosh (2001), hep-th/0109168.

[34] R. Kallosh, A. D. Linde, S. Prokushkin, and M. Shmakova, Phys. Rev. D65, 105016 (2002), hep-th/0110089.

[35] G. R. Dvali, Q. Shafi, and R. K. Schaefer, Phys. Rev. Lett. 73, 1886 (1994), hep-ph/9406319.

[36] H. V. Peiris et al. (2003), astro-ph/0302225.

[37] M. Colless et al. (2001), astro-ph/0106498.

[38] W. J. Percival et al. (2001), astro-ph/0105252.

[39] R. A. C. Croft et al., Astrophys. J. 581, 20 (2002), astro-ph/0012324.

[40] N. Y. Gnedin and A. J. S. Hamilton (2001), astro-ph/0111194.

[41] N. Arkani-Hamed, H.-C. Cheng, P. Creminelli, and L. Randall (2003), hep-th/0301218. 